\title{
Immune Response Regulation Pathway
}

National Cancer Institute

\section{Source}

National Cancer Institute. Immune Response Regulation Pathway. NCI Thesaurus. Code C39698.

An elaboration of the sequence of events that play a role in org anismal defense responses. 